\title{
Comparative evaluation of methods for isolating small extracellular vesicles derived from pancreatic cancer cells
}

\author{
Jie-Min Wang ${ }^{1,2,3+}$, Yong-Jiang $\mathrm{Li}^{1,2,3 \dagger}$, Jun-Yong Wu ${ }^{1,2,3}$, Jia-Xin Cai ${ }^{1,2,3}$, Jing Wen ${ }^{1,2,3}$, Da-Xiong Xiang ${ }^{1,2,3^{*}}$ (D), \\ Xiong-Bin $\mathrm{Hu}^{1,2,3}$ and Wen-Qun $\mathrm{Li}^{1,2,3}$
}

\begin{abstract}
Background: Small extracellular vesicles (sEVs) are nanosized vesicles involved in cell-to-cell communication. sEVs have been widely studied for clinical applications such as early detection of diseases and as therapeutics. Various methods for sEVs isolation are been using, but different methods may result in different qualities of sEVs and impact downstream analysis and applications. Here, we compared current isolation methods and performed a comparative analysis of sEVs from supernatant of cultured pancreatic cancer cells.
\end{abstract}

Methods: Ultracentrifugation, ultrafiltration and co-precipitation as concentration methods were firstly evaluated for yield, size, morphology and protein level of pellets. Then, isolate sEVs obtained by four different purification methods: size exclusion chromatography, density gradient ultracentrifugation, ultracentrifugation, and immunoaffinity capturing, were analysed and compared.

Results: For the concentration process, ultracentrifugation method obtained high quality and high concentration of pellets. For the purification process, immunoaffinity capturing method obtained the purest sEVs with less contaminants, while density gradient ultracentrifugation-based method obtained sEVs with the smallest size. Proteomic analysis revealed distinct protein contents of purified sEVs from different methods.

Conclusions: For isolating sEVs derived from supernatant of cultured pancreatic cancer cell line, ultracentrifugationbased method is recommended for concentration of $\mathrm{sEVs}$, density gradient ultracentrifugation-based method may be applied for obtaining purified sEVs with controlled size, immunoaffinity capturing may be suitable for studies requiring sEVs with high purity but may loss subtypes of sEVs without specific protein marker.

Keywords: Extracellular vesicles, Isolation method, Ultracentrifugation, Immunoaffinity capturing

\section{Introduction}

Extracellular vesicles (EVs) are biological vesicles released by almost all types of cells. EVs have gained increasing interests over the last decade for their cell-to-cell communication properties. EVs have been emerging as

\footnotetext{
*Correspondence: xiangdaxiong@csu.edu.cn

${ }^{\dagger}$ Jie-Min Wang and Yong-Jiang Li contributed equally to this work

1 Department of Pharmacy, The Second Xiangya Hospital, Central South University, Changsha, Hunan, China

Full list of author information is available at the end of the article
}

attractive therapeutic tools for their content and their natural carrier role. Small EVs are able to be engineered as nano drug delivery vehicles due to their relatively small size and properties such as crossing the biological barrier, circulation stability and inherent targeting.

The methods for isolation of sEVs has been extensively studied. Conventional differential ultracentrifugation has been widely used, but may not be able to remove all contaminants $[1,2]$. To obtain sEVs with small size, $0.22 \mu \mathrm{m}$ filtration membranes were used in some studies to remove the microvesicles [3, 4], but the filtration 
membrane could not remove protein contaminants, which may mislead the study results $[5,6]$. The International Society for Extracellular Vesicles (ISEV) advised that the qualities of EV from different isolation methods were different [7]. Besides, each isolation method may have disadvantages. Recently, it was reported that ultracentrifugation could not obtain EVs with high purity [1, 2], and may have problems such as clogging and trapping of vesicles [8], though the ultracentrifugation method could be improved by using a cushion of iodixanol [9]. Polymer co-precipitation-based methods are simple to perform but will precipitate large vesicles and contaminant proteins in the sample [10,11]. Size exclusion chromatography-based method and density gradient ultracentrifugation-based may be effective but could also be time-consuming [12]. Application of immunoaffinity capture-based method was limited by target selection [13].

It seems to be reasonable to select the isolation method according to the demand for EV qualities. Generally, EV biomarker study demanded the purest EVs for exploring the relationship between EVs and diseases [14, 15], and EV therapeutic study demanded pure and large quantities of EVs [16]. Pancreatic cancer-derived sEVs have shown potentials for early disease detection [17] and therapeutic application [18]. However, there has been a lack of comparison of isolation methods of sEVs derived from pancreatic cancer cells.

This study aims to evaluate methods for isolation of pancreatic cancer-derived sEVs. A two-step isolation process was performed: concentration and purification. The concentration step aims to concentrate EVs from conditioned cell culture medium, while the purification step aims to purify crude sEVs. Human pancreatic Panc-1 cells, a widely used cell line for pancreatic cancer model, was used for production of sEVs [19]. A details isolation process of sEVs from Panc-1 cells was shown in Fig. 1.

\section{Methods \\ Cell culture}

The Panc-1 cell line was obtained from the ScienCell (USA). Cells were maintained in DMEM (Dulbecco's modified Eagle's medium, Thermo Fisher Scientific, USA) supplemented with $10 \% \mathrm{EV}$-depleted fetal bovine serum (obtained via ultracentrifugation at $160,000 \mathrm{~g}$ for $12 \mathrm{~h}$ ) (Thermo Fisher Scientific, USA). Cells were incubated at $37^{\circ} \mathrm{C}$ with $5 \% \mathrm{CO}_{2}$.

\section{Preparation of $\mathrm{sEV} s$-containing medium}

Before sEVs isolation, the supernatant from Panc-1 cells was handled as previously described [20]. Briefly, supernatant was centrifuged at $300 \mathrm{~g}$ for $10 \mathrm{~min}$ to remove cells, centrifuged at $2000 \mathrm{~g}$ for $10 \mathrm{~min}$ to remove the dead cells, and then centrifuged at 10,000 g (Thermo Fisher ST 16R, USA) for $1 \mathrm{~h}$ to remove the cell debris and microvesicles [21]. The remaining supernatant was sEVs-containing medium.

\section{Preparation of crude sEVs}

The following methods were separately used for preparation of crude sEVs (Fig. 1a): (1) Ultracentrifugation (UC): $50 \mathrm{ml}$ of sEVs-containing medium was centrifuged at 100,000 g (SW32Ti, Beckman Coulter XPN-100 Ultracentrifuge, USA) for $70 \mathrm{~min}$ at $4{ }^{\circ} \mathrm{C}$, and the pellet was resuspended in $200 \mu \mathrm{L}$ of PBS [21]; (2) Ultrafiltration (UF): $50 \mathrm{ml}$ of sEVs-containing medium was concentrated using a $100 \mathrm{kDa}$ ultrafiltration centrifugal tube (Merck Millipore, USA), PBS was added to re-suspend the concentrate, resulting in a final volume of $200 \mu \mathrm{L}$ [22]; (3) Co-precipitation (Co-P): $50 \mathrm{~mL}$ of 8\% PEG 6000 (Solarbio, China) solution was prepared and then mixed with $50 \mathrm{ml}$ of sEVs-containing medium at $4{ }^{\circ} \mathrm{C}$ for $12 \mathrm{~h}$, and then centrifuged at $4000 \mathrm{~g}$ for $60 \mathrm{~min}$. The pellet was resuspended in $200 \mu \mathrm{L}$ of PBS. Crude sEVs were stored at $-80^{\circ} \mathrm{C}$ (within 2 days.) before further purification.

\section{Purification of sEVs}

Based on the results of evaluation of pellets after concentrating sEVs, ultracentrifugation was used for concentrating sEVs for further purification of sEVs. Four methods were used for purifying crude sEVs (Fig. 1b): (1) Density gradient ultracentrifugation (DGUC): $200 \mu \mathrm{L}$ of crude sEVs was loaded onto the top of a $12 \mathrm{~mL}$ discontinuous sucrose (Solarbio, China) gradient solution $(15 \%, 20 \%$, $25 \%, 30 \%, 40 \%, 60 \%$ sucrose in PBS, $2 \mathrm{~mL}$ for each gradient solution) and then centrifuged at 100,000 $\mathrm{g}$ for $16 \mathrm{~h}$ at $4{ }^{\circ} \mathrm{C} .12$ fractions $(1 \mathrm{ml})$ were collected for each gradient [23]. (2) Size exclusion chromatography (SEC): Sepharose CL-2B (Solarbio, China) was loaded into an injector (10 $\mathrm{mL}$ ), with cotton blocked at the bottom. $200 \mu \mathrm{L}$ of crude sEVs was loaded onto the top of the column. Then the column was eluted by PBS, and each $1 \mathrm{~mL}$ of eluate was collected for 12 sequential fractions [24]. (3) UC: 200 $\mu \mathrm{L}$ of crude sEVs was washed in PBS. Then the solution was centrifuged at $10,000 \mathrm{~g}, 4{ }^{\circ} \mathrm{C}$ for $70 \mathrm{~min}$. The pellet was re-suspend by $200 \mu \mathrm{L}$ of PBS. (4) Immunoaffinity capture (IAC): Magnetic beads (BeaverBeadsTM Protein A/G immunoprecipitation kit, Beaver, China) were washed and then activated by incubating with $100 \mu \mathrm{L}$ of anti-CD63 antibody (50 $\mu \mathrm{g} / \mathrm{mL}$, ab134331, abcam, UK) for $15 \mathrm{~min}$. After magnetic separation, the anti-CD63conjugated beads were incubated with $200 \mu \mathrm{L}$ of crude sEVs at $25{ }^{\circ} \mathrm{C}$ for $1 \mathrm{~h}$. The sEVs-beads complexes were separated by a magnet and eluted and re-suspended in 40 $\mu \mathrm{L}$ of PBS. Purified sEVs were stored at $-80^{\circ} \mathrm{C}$ within two days before analysis. 

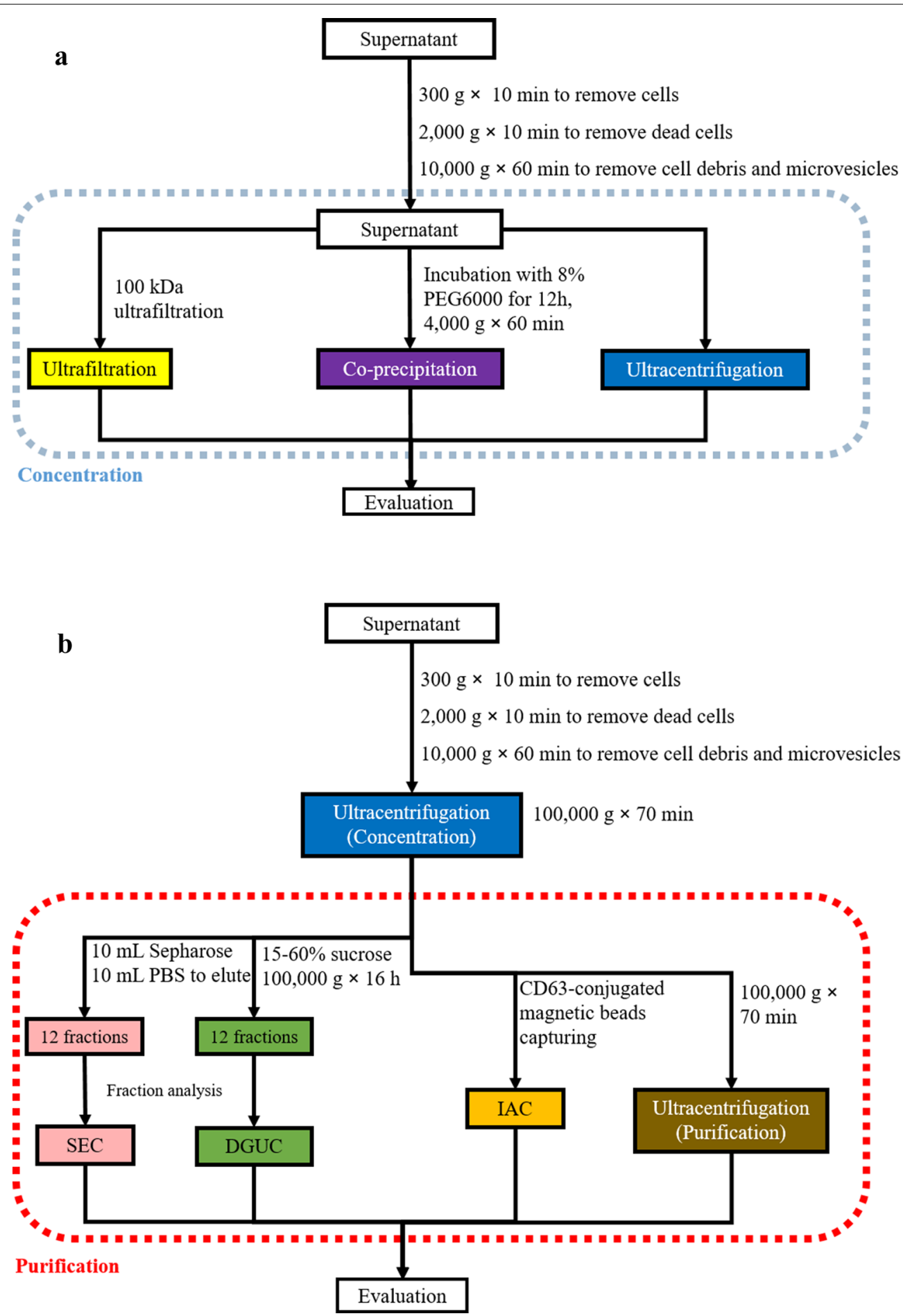

Fig. 1 The workflow of the study. a Three different methods for concentrating sEVs from cell culture medium. $\mathbf{b}$ Four different methods for purification of sEVs after standard differential ultracentrifugation

\section{Analysis of fraction from DGUC and SEC}

Total protein level in each fraction was determined by using a BCA Protein Assay Kit (MultiSciences Biotech
Co., China). The level of CD63 (CSB-E14107h) and CD9 (CSB-EL004969HU) in each fraction was determined by using an enzyme-linked immuno-sorbent assay (ELISA) 
kit (CUSABIO Biotech Co. Ltd., China). After analysis, fractions 6, 7, 8, 9, 10 from DGUC were mixed used for further analysis, while fractions 9, 10 from SEC were mixed and used for further analysis.

\section{Characterization and analysis of crude sEVs and purified sEVs \\ Nanoparticle tracking analysis (NTA)}

For crude sEVs, samples were diluted 100 times with PBS before NTA. For purified sEVs, samples obtained via IAC was diluted 125 times, samples obtained via SEC was diluted 2.5 times, samples obtained via UC was diluted 25 times, while samples obtained via DGUC was not diluted before NTA (NS300, Malvern, UK). The RR of particles was calculated as the following formula. The assay was repeated 3 times.

$$
R R \text { of particles }=\frac{\text { number of particles in purified } s E V s}{\text { number of particles in crude } s E V s}
$$

\section{Transmission electron microscopy}

$10 \mu \mathrm{L}$ of samples was dropped onto an ultrathin carbon film-coated 400 mesh copper grid and washed with PBS for two times. After drying excess liquid, the EVs-coated grid was stained by phosphotungstic acid (1\%) and then washed with PBS for two times and then dried and imaged with a multipurpose field emission transmission electron microscope (TEM, JEM-1200EX, JEOL Ltd., Japan).

\section{Protein level}

The protein level was determined by using a BCA Protein Assay Kit. The Protein Recovery rate (RR) of the purification process was calculated as the following formula. The assay was repeated 3 times.

$$
R R \text { of protein }=\frac{\text { Total protein in purified } s E V s}{\text { Total protein in crude } s E V s}
$$

\section{Western blotting}

$5 \times$ SDS-PAGE Loading Buffer (New cell and Molecular Biotech Co., Ltd, China) was added into the sample. The sample was kept at $100{ }^{\circ} \mathrm{C}$ for $10 \mathrm{~min} .6 .02 \times 10$ [9] EVs were loaded on each well in 12\% SDS-PAGE (Lianke Bio, China). After the electrophoresis, the proteins were transferred to PVDF membrane (Millipore, USA). The membrane was blocked with $5 \%$ milk solution for 1.5 $\mathrm{h}$, followed by incubation with anti-CD63 (ab134045, abcam, UK), anti-CD81 (GB111073, Servicebio, China), anti-CD9 (ab92726, abcam, UK), anti-CD47 (ab108415, abcam, UK), anti-GAPDH (AF7201, Affinity, China), and anti-ago 1(\#9388, Cell Signaling, USA). The PVDF membrane was washed and then incubated with Peroxidase-conjugated Goat anti-Rabbit IgG (ZSGB-Bio, China). The membrane was incubated with ECL luminescent (New cell and Molecular Biotech Co., Ltd, China) for 3 min for detection.

\section{Coomassie brilliant blue staining}

$5 \times$ SDS-PAGE Loading Buffer (New cell and Molecular Biotech Co., Ltd, China) was added into the sample. The sample was kept at $100{ }^{\circ} \mathrm{C}$ for $10 \mathrm{~min} .6 .02 \times 10^{9} \mathrm{EVs}$ were loaded on each well in 12\% SDS-PAGE (Lianke Bio, China). After the electrophoresis, the gel was incubated with $20 \mathrm{~mL}$ of working solution $(0.0025 \%$ Coomassie brilliant blue, 45\% methanol, 10\% glacial acetic acid) (Solar bio, China) for $1 \mathrm{~h}$, and then washed by elution solution (25\% methanol, $8 \%$ glacial acetic acid) for $4 \mathrm{~h}$.

\section{ELISA}

The level of CD63, CD81, TSG101, beta-actin, GAPDH, CD47 (CUSABIO Biotech Co. Ltd., China) and ago-1 (MyBiosource, Canada) in purified samples and in crude sEVs were determined by ELISA kits. The protein per EV was calculated by the following formula. The assay was repeated 3 times.

$$
\text { Protein per } E V=\frac{\text { total protein }}{\text { number of } E V S}
$$

\section{Digestion of proteins}

SDT solution (4\% SDS, $100 \mathrm{mM}$ Tris- $\mathrm{HCl}, \mathrm{pH}$ 7.6) was added into the purified sEVs. The sample was incubated under boiling water for $15 \mathrm{~min}$, followed by centrifugation at $14,000 \mathrm{~g}$ for $15 \mathrm{~min}$. The supernatant was collected as protein sample. DTT (Sigma, USA, 43819-5G) was added in the protein sample to $100 \mathrm{mM}$. The sample was incubated under boiling water for $5 \mathrm{~min}$, and then cooled to room temperature. $200 \mu \mathrm{L}$ of UA buffer $(8 \mathrm{M}$ Urea, $150 \mathrm{mM}$ Tris- $\mathrm{HCl}, \mathrm{pH} 8.5$ ) was added to the sample, followed by centrifuging at $12,500 \mathrm{~g}$ for $15 \mathrm{~min}$ using a $30 \mathrm{kDa}$ ultrafiltration tube and centrifuged at $12,500 \mathrm{~g}$ for $15 \mathrm{~min}$. Then, $100 \mu \mathrm{L}$ of iodoacetamide (IAA) buffer (100mM IAA in UA) was added and kept at room temperature in darkness for $30 \mathrm{~min}$. The sample was centrifuged at $12,500 \mathrm{~g}$ for $15 \mathrm{~min}$. $100 \mu \mathrm{L}$ of UA buffer was added to the supernatant and then centrifuged at 12,500 g for 15 min again. $100 \mu \mathrm{L}$ of $40 \mathrm{mM} \mathrm{NH}_{4} \mathrm{HCO}_{3}$ solution was added to the sample followed by centrifugation at $12,500 \mathrm{~g}$ for $15 \mathrm{~min}$. Then, $40 \mu \mathrm{L}$ of Trypsin buffer was added ( $4 \mu \mathrm{g}$ Trypsin in $40 \mu \mathrm{L}$ of $40 \mathrm{mM} \mathrm{NH}_{4} \mathrm{HCO}_{3}$ ) to the sample and incubated at $37^{\circ} \mathrm{C}$ for $16-18 \mathrm{~h}$. The sample (in a filtration tube) was centrifuged at $12,500 \mathrm{~g}$ for $15 \mathrm{~min}$, then $20 \mu \mathrm{L}$ of $40 \mathrm{mM} \mathrm{NH}_{4} \mathrm{HCO}_{3}$ solution was added and centrifuged at 12,500 for 15 min to obtain the filtrate. A 
C18 cartridge (WAT023590, Waters) was used to desalinate. After freeze-drying, $40 \mu \mathrm{L}$ of $0.1 \%$ methanol solution was added to the solid to re-solute the sample.

\section{LC-MS/MS}

Then sample was separated by Easy nLC (Thermo Fisher Scientific, USA). Solution A: 0.1\% FA; Solution B: 0.1\% FA, 80\% ACN. Chromatographic column (Acclaim PepMap RSLC $50 \mu \mathrm{m} \times 15 \mathrm{~cm}$, nano viper, P/N164943, Thermo Fisher Scientific) was balanced by $100 \%$ solution A. Velocity of flow was $300 \mathrm{~nL} / \mathrm{min}$. Gradient elution: 0-5 min, solution B 3\%; 5-45 min, solution B 3\%-28\%; 45-55 min, solution B $28 \%-38 \%$; $50-55$ min, solution B $38 \%-100 \%$; 55-60 min, solution B 100\%.

The sample was analysed by Q Exactive (Thermo Fisher Scientific, USA). Analysis time was $1 \mathrm{~h}$. The mode was positive ion mode. Range of parent ion was $350-1800$ $\mathrm{m} / \mathrm{z}$. The resolution of mass spectrometry was 7000 . AGC target was $3 \mathrm{e}^{6.1}$ stage Maximum IT was $50 \mathrm{~ms}$. Via full scan 10 MS2 scans were acquired. MS2 Activation Type was HCD. Isolation window was $2 \mathrm{~m} / \mathrm{z}$. The resolution of 2 stage mass spectrometry was 17,500. Microscan was 1.2 stage. Maximum IT was $45 \mathrm{~ms}$. Normalized Collision Energy was 27eV.

Data-dependent acquisition was performed. The peptides database was Uniprot_HomoSapiens_20386_20180905, downloaded in http://www.unipr ot.org. MaxQuant 1.5.5.1 was used to qualitative analysis. Label Free Quantitation was used for quantitative analysis.

\section{Statistical analysis}

Data were presented as mean values \pm SD. One-way analysis of variance (ANOVA) and students' $t$ test were performed at the significance level $\alpha=0.05$.

\section{Results}

\section{Analysis of crude sEVs}

Same volume $(50 \mathrm{ml})$ of the supernatant was processed to compare concentration methods. Size distribution of crude sEVs obtained via UF, UC and Co-P were presented in Fig. 2a, b and c, respectively. sEVs obtained via UC showed a smaller size distribution than other two methods. All samples showed plenty of big particles, indicating that neither single method (UC, Co-P or UF) could obtain pure sEVs. Crude sEVs obtained via UC showed significantly more total particle number and small particle (30-150nm) number than UF and Co-P (Fig. 2d). sEVs obtained via UF showed significantly high protein levels than UC and Co-P as evaluated by BCA assay test (Fig. 2e) and coomassie brilliant blue staining (Fig. 2f), but the protein level in the control (fresh medium after UC) was also very high. TEM images for crude sEVs were presented in Fig. 2g. Big and small EVs could be observed for all crude sEVs groups. Besides, aggregation of unknown small particles (red frame) was found in crude sEVs obtained via Co-P. Based on the quality of crude sEVs, UC was used for crude sEVs collection for further purifications.

\section{Analysis of fraction from DGUC and SEC}

Total protein, CD63 and CD9 levels in fractions from DGUC and SEC were shown in Fig. 3. Fractions with a relatively high level of CD63 and CD9 were collected as purified sEVs $[24,25]$. CD63 and CD9 showed consistent levels in most fractions. Hence, fractions 6, 7, 8, 9 and 10 from DGUC were mixed as the purified sample (Figure $3 a, b)$, fractions 9 and 10 from SEC were mixed as the purified sample (Figure 3c, d).

\section{Analysis of purified sEVs}

The size distribution of purified samples via UC, SEC, IAC and DGUC were shown in Figure 4a-d, respectively. The big particles in sEVs for all samples were removed substantially. The size of the sample via DGUC showed the smallest size, and the sample via IAC showed a relatively small size with less microvesicles. The sample via UC showed more particle and sEVs numbers than other methods (Figure 4e). The sample via SEC showed the least particles and sEVs. Besides, samples via DGUC or IAC showed a relatively high proportion of sEVs in all particles (Figure 4e). The sample via UC showed the highest RR (Figure 4f). TEM images of purified sEVs were shown in Figure 4g. sEVs could be observed for all samples, while samples obtained via UC show relatively more particles. Samples obtained via IAC and DGUC showed clearer background under TEM. Contaminants were observed in samples obtained via SEC.

\section{Protein evaluation}

Purified sEVs showed lower total protein levels for all methods than crude sEVs (Figure 5). The protein level of the sample via UC was higher than other methods as evaluated by BCA assay (Figure $5 \mathrm{c}$ ) and coomassie brilliant blue staining (Figure 5a). The sample purified by IAC showed the least total protein level and recover rate (Figure 5c, d).

WB results were shown in Figure 5b. Three EVs marker proteins (CD81, CD63, CD9) and CD47 were detected in the purified samples. Compared to the crude sEVs, ago-1 as contaminant protein was significantly decreased in purified samples, which was consistent with the WB results (Figure 5e). Results of ELISA were summarized in Figure 5f. Samples purified by UC showed less CD47, CD81, GAPDH and $\beta$-actin. The sample via IAC showed the least TSG101. The sample via IAC had a higher CD63 
a

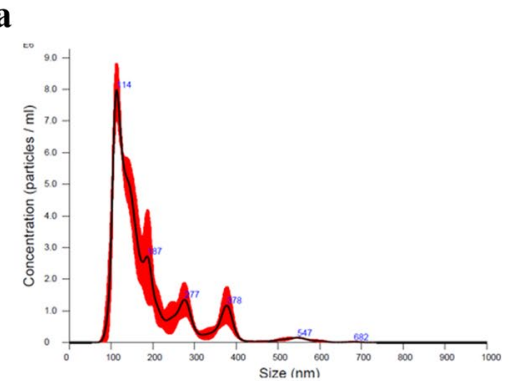

d

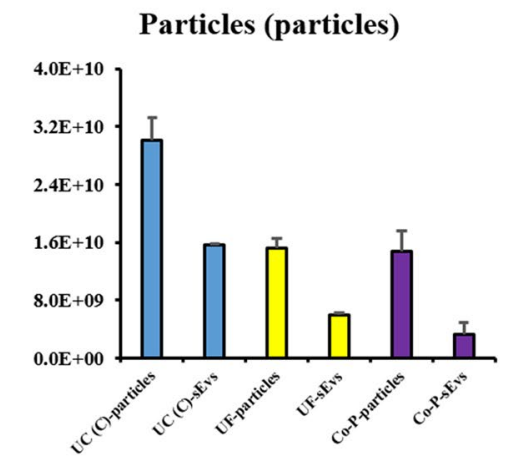

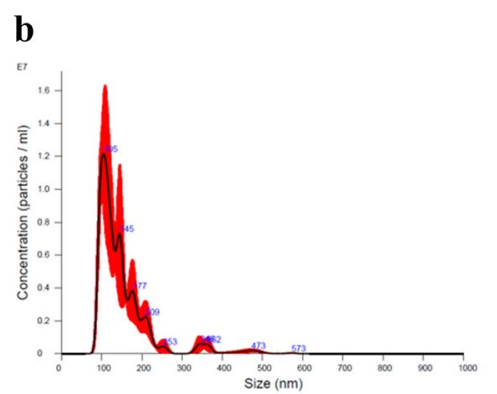

e

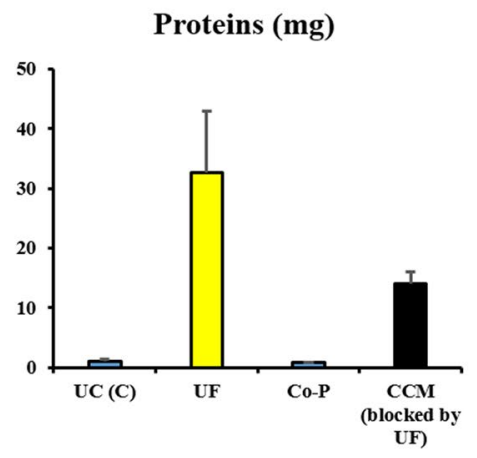

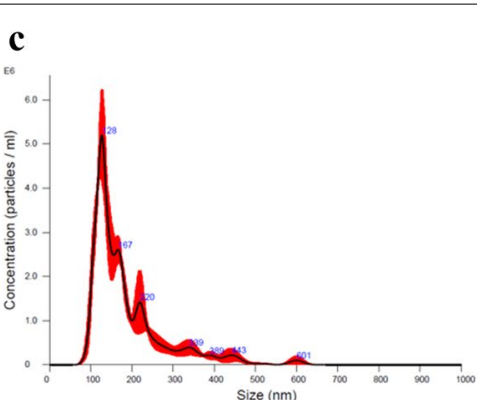

f

g
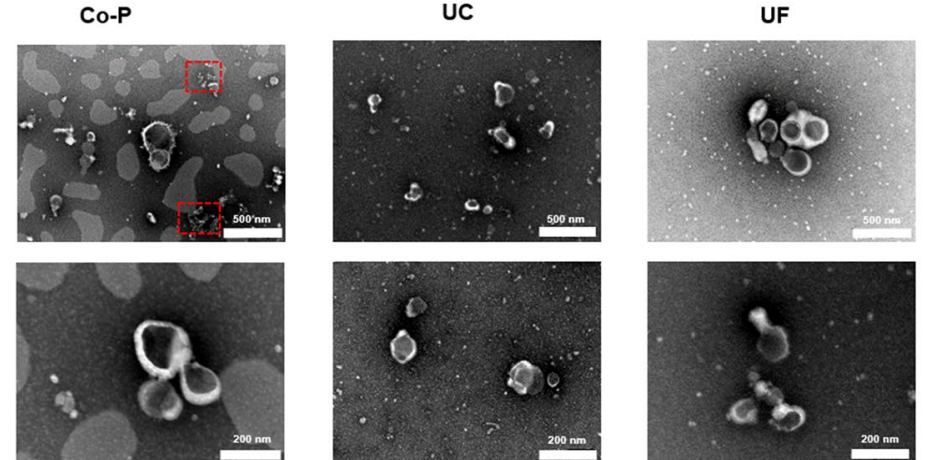

Fig. 2 Evaluation of crude sEVs obtained via three different concentration methods. a Size distribution of pellets via ultrafiltration $(n=3)$. b Size distribution of pellets via ultracentrifugation $(n=3)$. c Size distribution of pellets via co-precipitation $(n=3)$. $\mathbf{d}$ The numbers of total particles and sEVs $(30-150 \mathrm{~nm})$ in the pellets $(n=3)$. e The yield of the protein in pellets after concentration process $(n=3)$. $\mathbf{f}$ Coomassie brilliant blue staining of pellet. $\mathbf{g}$ TEM images of pellets. Contaminants of aggregated small particles (red frame) could be observed in the pellet obtained via co-precipitation method

level as the sample was isolated by anti-CD63-conjugated beads. RR of CD63 was almost $100 \%$ for IAC method, indicating that almost all particles expressing CD63 were extracted. For the samples purified by SEC and DGUC, levels of most proteins tested were similar.

\section{Proteomics}

A total of 817 proteins were detected in proteomic study. There were 631 proteins in the crude sEVs sample via UC, 383 proteins in the purified sEVs sample via UC, 78 proteins in the sample via SEC, 154 proteins in the sample via DGUC and 76 proteins in the sample via IAC, 25 proteins were identified for all five groups (Fig. 6a). Heat map analysis of all proteins was summarized in Figure 6b. Purified sEVs obtained via UC showed significantly more protein content, which was consistent with our results of protein evaluation (Fig. 5). Reported potential protein biomarkers for cancer development [26], metastasis [27] and drug resistance [28] were also analysed in our proteomic study. Further analysis revealed distinct protein contents in samples (Fig. 6). High contents of both overexpressed and downexpressed proteins associated with 
$\mathbf{a}$

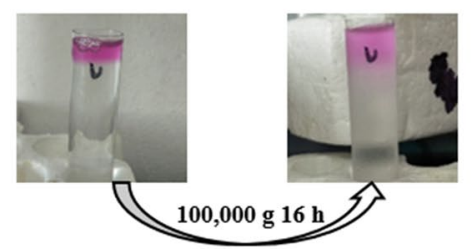

c

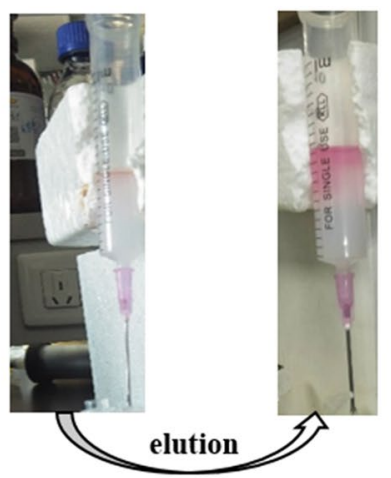

b

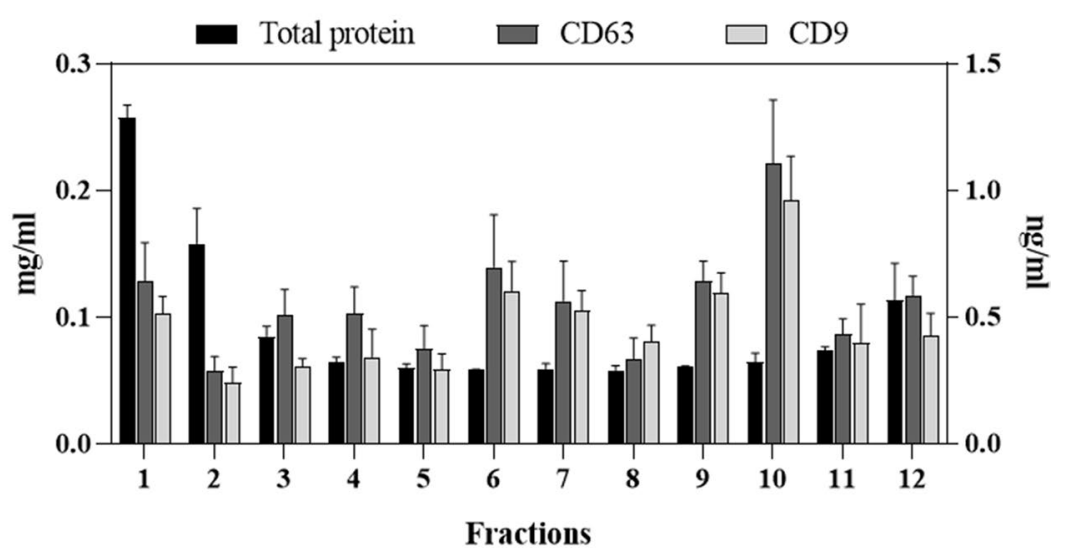

d

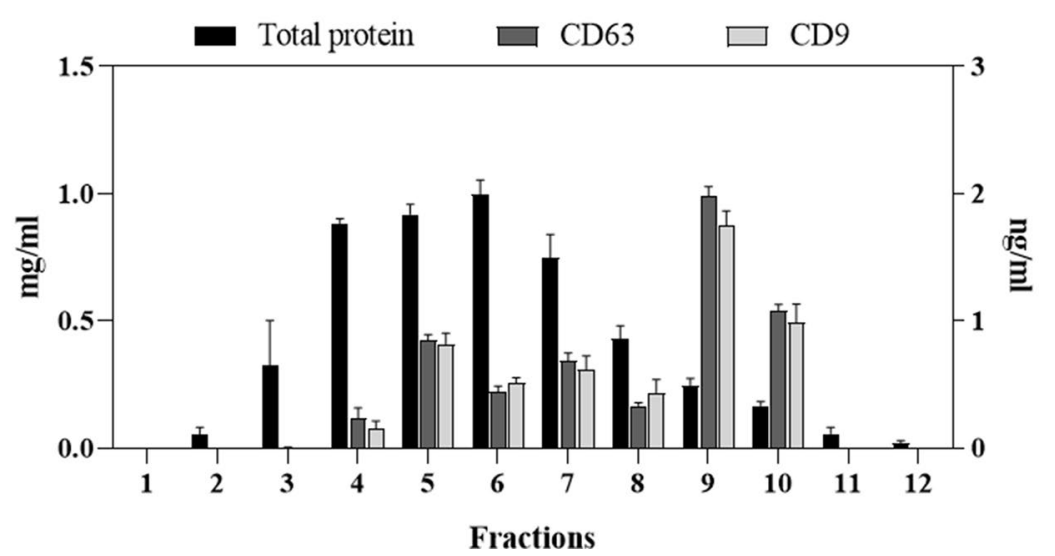

Fig. 3 SEC purification process of the pellet obtained via ultracentrifugation. a The picture of the ultracentrifuge tube before and after ultracentrifugation. The border of the concentrate and ultracentrifugation rose solution became vague after ultracentrifugation. $\mathbf{b}$ Fraction analysis of DG ultracentrifugation $(n=3)$. Because of high CD63 concentration, the fraction $6,7,8,9$ and 10 were merged as the purified sample. c The picture of SEC, the EVs concentrate was eluted. $\mathbf{d}$ Fraction analysis of SEC $(n=3)$. Because of high CD63 concentration, the fraction 9 and 10 were merged as the SEC purified sample. ${ }^{*} \mathrm{P}<0.05$. Red color indicates the cell culture supernatant

metastasis and drug-resistance were detected in crude sEVs (Fig. $6 \mathrm{c}$ to h). Purified sEVs via IAC retained most pancreatic cancer-overexpressed (Fig. 6c), but not downexpressed (Fig. 6d), proteins (compared to normal adjacent pancreatic tissue). sEVs obtained via SEC showed the least contents of proteins associated with metastasis (Fig. 6e) and drug resistance (Fig. 6g).

\section{Discussion}

Despite progress in technique, isolation of sEVs has been challenging. It would be wise to choose a strategy and develop a isolation specific protocol depending on experiment purpose. Three concentration methods and four purification methods for obtaining sEVs from cultured Panc-1 cells were evaluated in our study. A summarization of advantages, disadvantages and possible suggestions for isolation methods used in this study was shown in Table 1. For the concentration of sEVs, UC-based method showed high yield and purity of sEVs and was used to concentrate the sample before purification. For the purification of sEVs, IAC was effective and time-saving and yield purest sEVs among all methods evaluated, thus may be suitable for study demanding high purity of sEVs $[14,15]$. DGUC method effectively produced the smallest and also purified sEVs with relatively high yield, thus may be suitable for obtaining sEVs with controlled size and large quantity [16, 29-31]. More importantly, sEVs obtained via DGUC method could avoid aggregation and precipitation [32] and were superior for cellular uptake [33].

In the presented study, the size, yield, morphology and protein were assessed for quality of sEVs. The size and yield of sEVs were crucial for therapeutic use, especially for drug delivery. It has been reported that smaller EVs could be uptaken by cells more efficiently [33]. The morphology showing the presence of EV was observed, sEVs obtained in this study was saucer-shaped under TEM images as reported [34]. CD63 was a marker for EVs and 


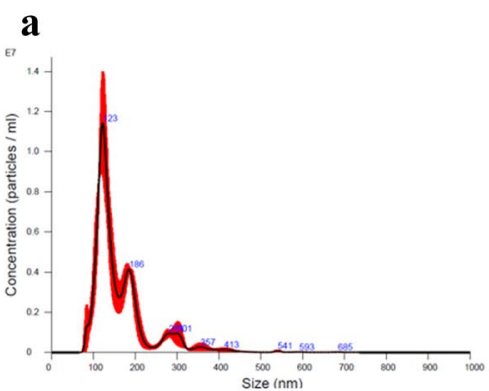

c

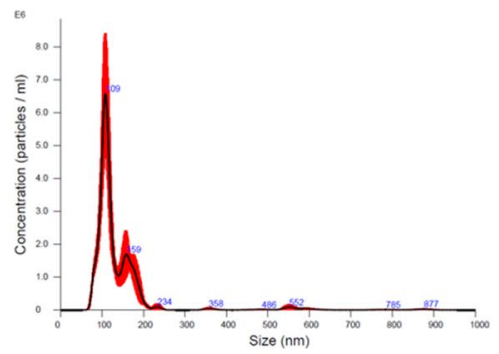

e

Particles (particles)

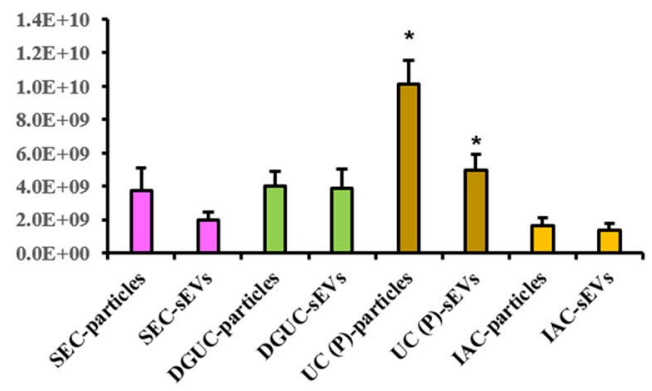

f

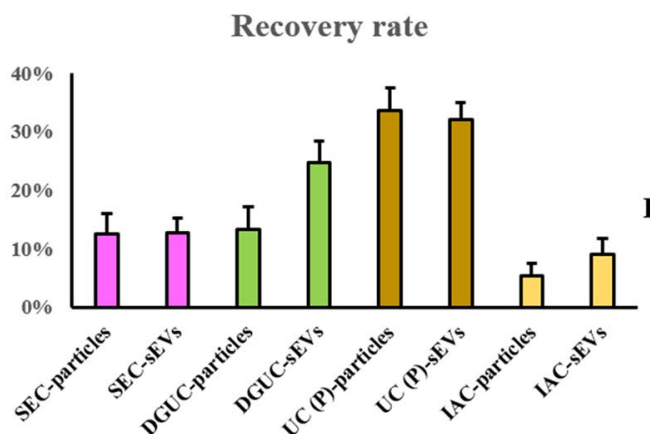

b

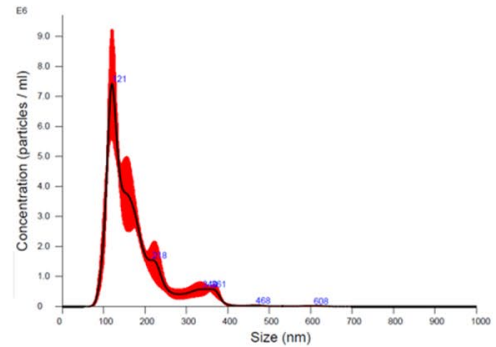

d

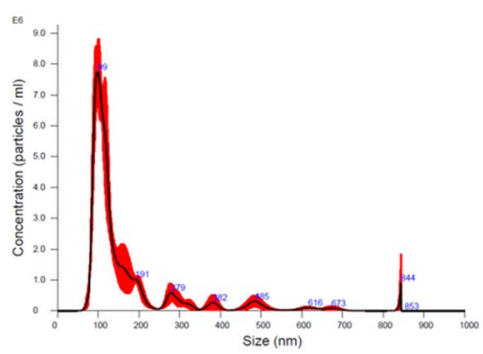

g
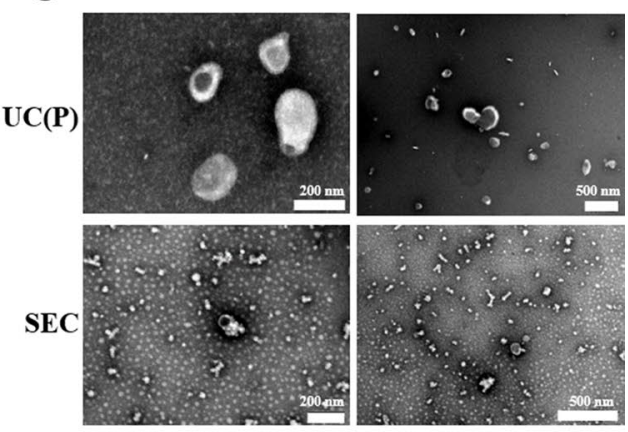

IAC

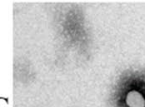

O

DGUC
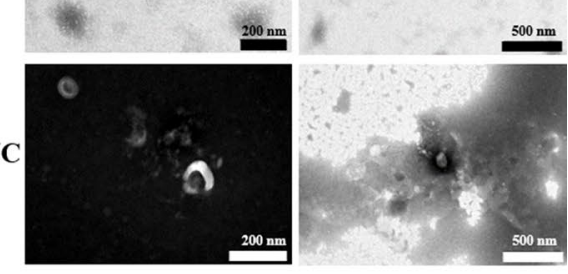

Fig. 4 Evaluation of particles of the purified samples. a Size distribution of pellets via ultracentrifugation $(n=3)$. $\mathbf{b}$ Size distribution of pellets via SEC $(n=3)$. c Size distribution of pellets via IAC $(n=3)$. c Size distribution of pellets via DGUC $(n=3)$. e The numbers of total particles and sEVs $(30-150$ $n m)$ in the pellets $(n=3)$. $\mathbf{f R R}$ of the particle and sEVs. $\mathbf{g}$ TEM images of the purified samples. e Dyed-gel. Compared of the concentrates, the purified samples showed lower protein content. ${ }^{*} \mathrm{P}<0.05$

was used in IAC for purification [10,35, 36]. Besides, in our fraction analysis, CD63 and CD9 levels were used to reflect EV-containing fractions, this method was also reported in previous studies [23, 25, 37]. However, for SEC, CD63 and CD9 may not fully represent the sEVs fractions as expected. Possibly, CD63 and CD9 were not 
a UC(P) SEC DGUC IAC Marker

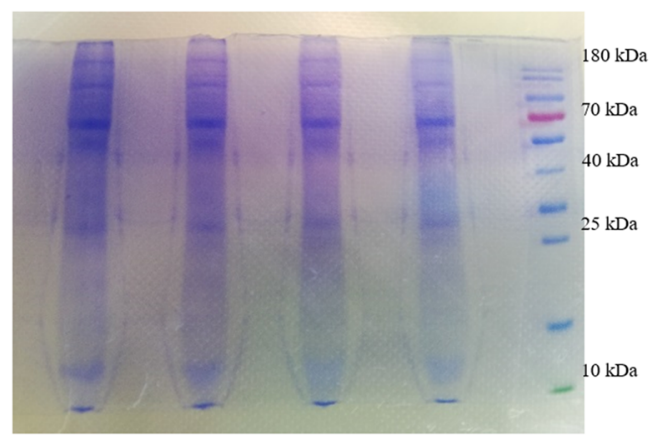

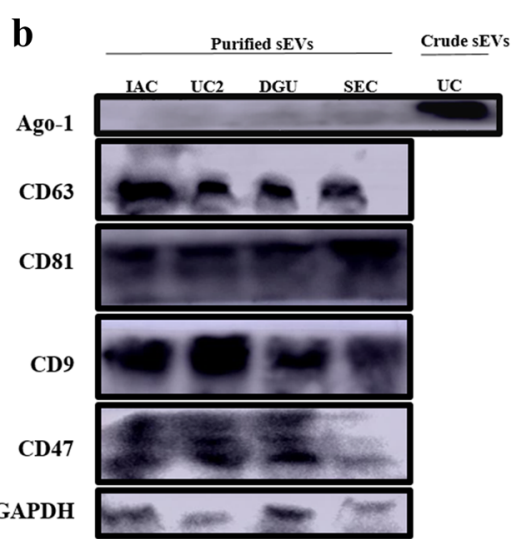

c

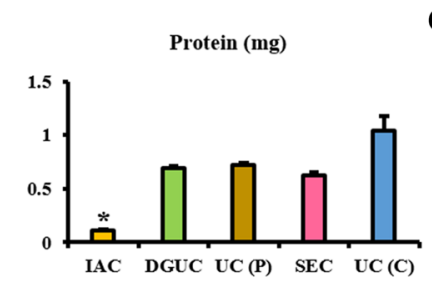

d

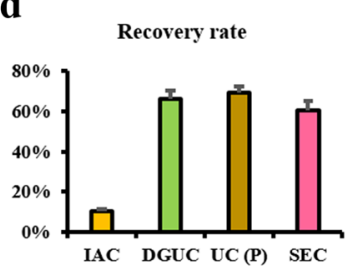

e

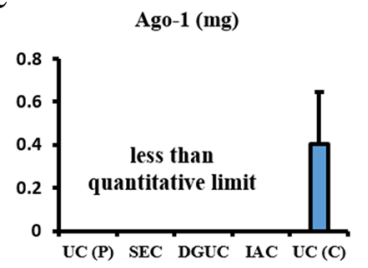

f

Average CD47 (pg/EV)

RR of CD47

Average CD81 (ng/EV)

RR of CD81
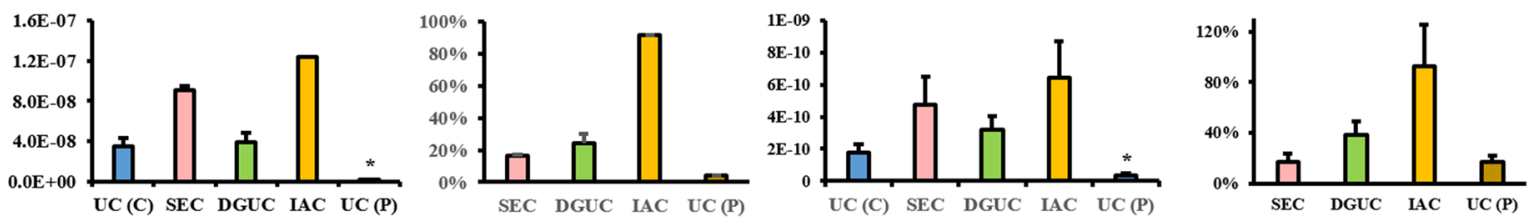

RR of $\beta$-actin
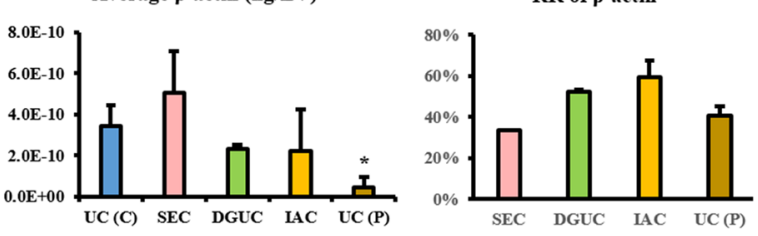

Average GAPDH (pg/EV)

RR of GAPDH

Average TSG 101 (pg/EV)

RR of TSG101

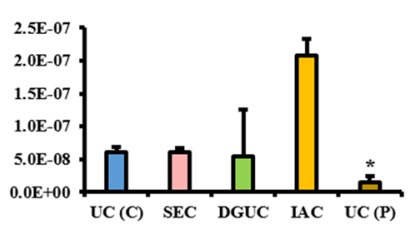

Average CD63 (ng/EV)
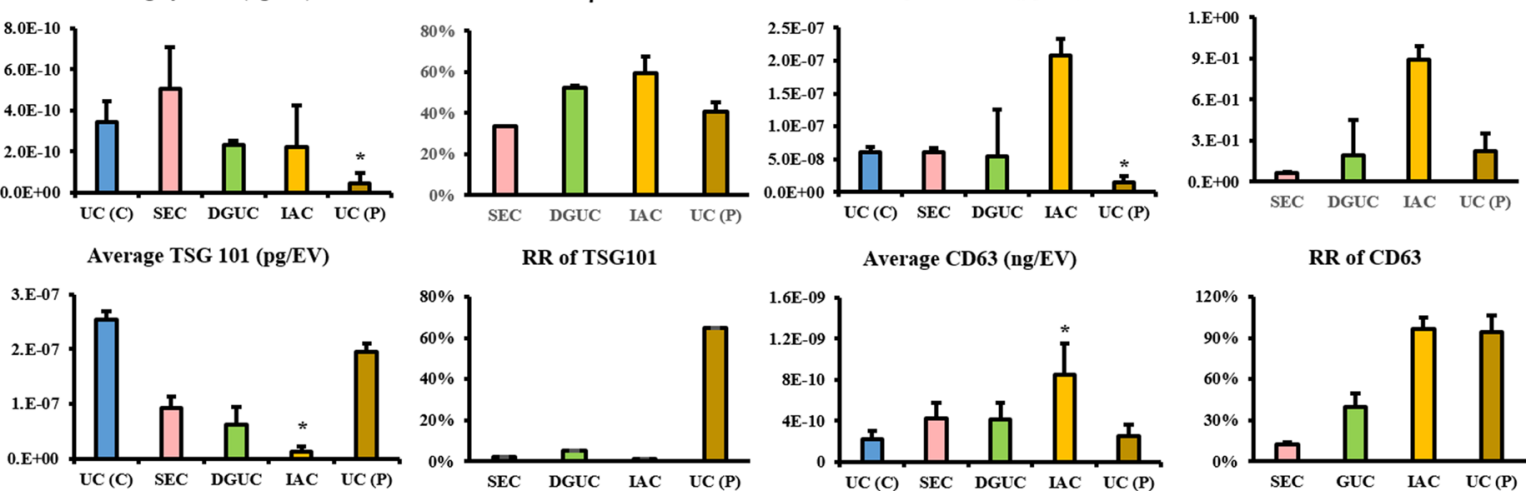

RR of CD63

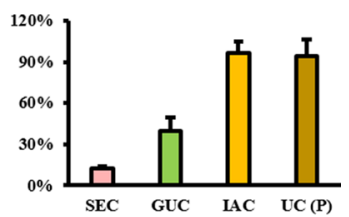

Fig. 5 Evaluation of proteins of the purified samples. a Coomassie brilliant blue staining of pellet. $\mathbf{b}$ Western blot analysis of purified sEVs sample. $\mathbf{c}$ Levels of protein in purified sample $(\mathrm{n}=3)$. $\mathbf{d}$ Recover rate of protein via different purification method. e Ago-1 level in purified samples. $\mathbf{f}$ Level of various proteins and recover rate in purified sample. ${ }^{*} \mathrm{P}<0.05$

exclusive to SEVs. Recent studies reported that microvesicles also expressed CD63 and CD9 [38, 39]. A combined strategy of more sEVs marker proteins such as TSG101 and CD81 may reflect the sEVs-containing fractions more accurately.

Our study included the majority of current sEVs isolation methods, except commercial kits and microfluidic-based methods. Because of unstable quality of the extracted EVs, high price and unknown solutions [40], commercial kits were not included in our study. The microfluidic technology was popular but not included in our study as the technique was mostly used for methodological studies, such as sEVs detection, 
$\mathbf{a}$

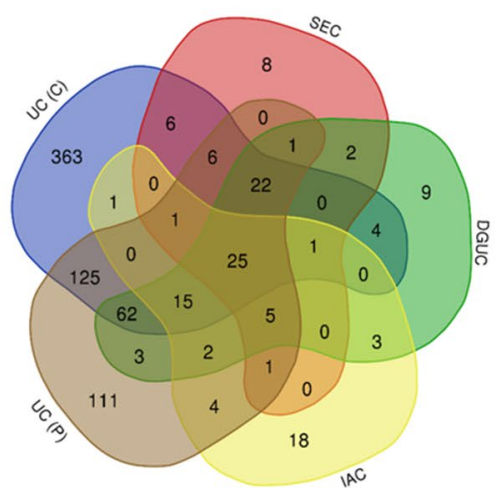

b
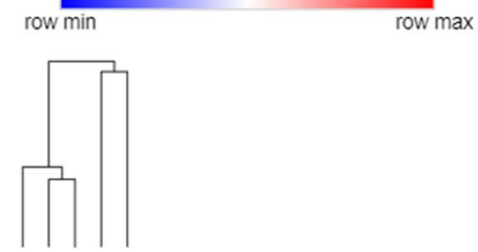

Qิe

id

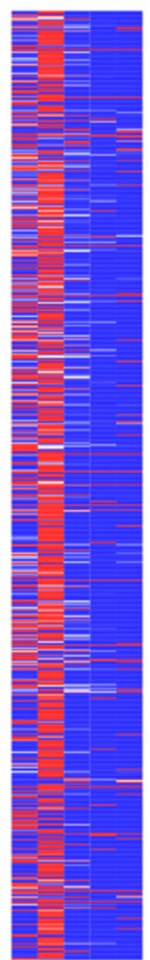

c

row min

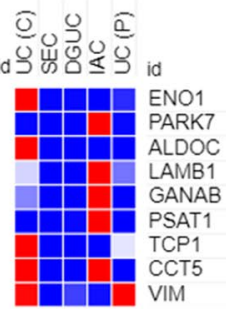

d

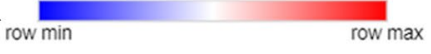

Q 0 id

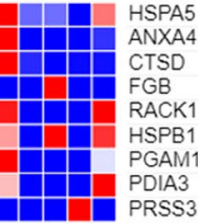

e

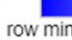

Q0

id $\mathcal{3}$ 岁政

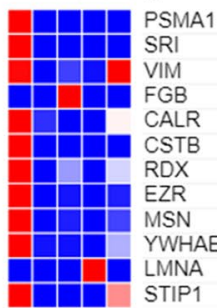

f

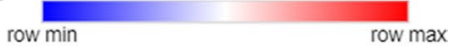

E. 0

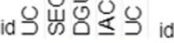

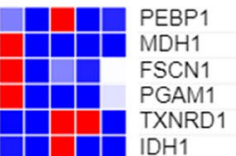

g row min

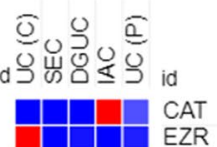

h

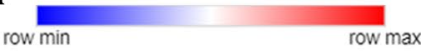

Q 00

id

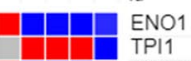

Fig. 6 Proteomic analysis of sEVs. a Venn diagram for number of proteins in sample. $\mathbf{b}$ The heat map of all proteins of $\mathrm{sEV} s(\mathrm{n}=3)$. $\mathbf{c}$ Analysis of overexpressed proteins (pancreatic cancer tissue compared with normal adjacent pancreatic tissue) in sEVs. $\mathbf{d}$ Analysis of downexpressed proteins (pancreatic cancer tissue compared with normal adjacent pancreatic tissue) in sEVs. e Analysis of overexpressed pancreatic cancer metastasis-associated proteins in sEVs. f Analysis of downexpressed pancreatic cancer metastasis-associated proteins in sEVs. $\mathbf{g}$ Analysis of overexpressed pancreatic cancer drug resistance-associated proteins in $\mathrm{SEV}$ s. $\mathbf{h}$ Analysis of downexpressed pancreatic cancer drug resistance-associated proteins in sEVs 
Table 1 Summary of concentration and purification methods for isolating sEVs from pancreatic cancer cells

\begin{tabular}{|c|c|c|c|c|c|}
\hline Process & Method & Advantages & Disadvantages & Possible suggestions & Recommendation \\
\hline \multirow[t]{3}{*}{ Concentration } & UC & High yield & High equipment cost & NA & $\begin{array}{l}\text { Suitable for concentrating } \\
\text { medium }\end{array}$ \\
\hline & $\mathrm{CO}-\mathrm{P}$ & Convenient & Non-exosomal contaminants & NA & NA \\
\hline & UF & Convenient & Non-exosomal contaminants & NA & NA \\
\hline \multirow[t]{4}{*}{ Purification } & UC & $\begin{array}{l}\text { High yield } \\
\text { Feasibility }\end{array}$ & High equipment cost & Fully re-suspend the crude sEVs & NA \\
\hline & SEC & $\begin{array}{l}\text { High purity } \\
\text { Up-scale isolation }\end{array}$ & $\begin{array}{l}\text { Require methodological valida- } \\
\text { tion }\end{array}$ & $\begin{array}{l}\text { Lengthen the column and } \\
\text { increase the sepharose } \\
\text { Slow down the flow rate } \\
\text { Lower the temperature }\end{array}$ & NA \\
\hline & DGUC & $\begin{array}{l}\text { Small EVs } \\
\text { High purity }\end{array}$ & $\begin{array}{l}\text { Require methodological study } \\
\text { Time-consuming centrifugation } \\
\text { High equipment cost }\end{array}$ & $\begin{array}{l}\text { Pilot test for exploring suitable } \\
\text { gradient solutions }\end{array}$ & Suitable for therapeutic study \\
\hline & IAC & $\begin{array}{l}\text { Small EVs } \\
\text { Time-saving } \\
\text { High purity }\end{array}$ & $\begin{array}{l}\text { Low yield } \\
\text { Subtype of sEVs }\end{array}$ & $\begin{array}{l}\text { Personalized customization } \\
\text { depending on aim of study }\end{array}$ & Suitable for biomarker study \\
\hline
\end{tabular}

Co-P, Co-precipitation; DGUC, Density gradient ultracentrifugation; EV, Extracellular vesicles; IAC, Immunoaffinity capture; NA, Not available; SEC, Size exclusion chromatography; UC, Ultracentrifugation; UF, Ultrafiltration

instead of therapeutic application study, even if it was potentially available for isolation [41, 42].

In the presented study, an equal number of EVs particles was evaluated for each method in case of contaminant protein influencing the results. The western blot analysis showed inconsistent intensity of GAPDH as cytosolic protein in sEVs, suggesting that different isolation methods would result in inconsistent purity and yield of sEVs and possibly subtype of sEVs. The level of target protein could be weak as detected if equal protein, with a high amount of contaminant protein, was loaded onto each well in the gel. This may explain why the level of EV marker protein was weak in study [43].

The UC-based technique remains the most common method for sEVs isolation [44-46]. However, our study results demonstrated that DGUC and IAC methods produced sEVs with better purity than UC. Besides, the interpretation of results should be cautious when commercial $\mathrm{sEVs}$ isolation kits based on the Co-P method was used [40,47]. The combination of several isolation methods may produce purer sEVs. Jeppesen et al. used ultracentrifugation-based technique, density gradient ultracentrifugation-based technique and immunoaffinity capture-based technique to purify sEVs [48]. But more isolation steps may produce fewer sEVs, and combined isolation protocols were often hard to follow as the strategy could be complicated and fussy. The technique for evaluation of sEVs has been advancing. Tian et al. splendidly used nanoflow cytometry to evaluate the quality of sEVs [40]. But nanoflow cytometry-based analysis of sEVs needs further refinement of methods. For better comparisons between groups and future replication, we applied basic characterization of sEVs isolated by different methods $[25,37,49]$.

sEVs are a heterogenous group of vesicles [50]. Single EV may carry distinct proteins, isolation method based on EV marker may lose EV subtypes of potential interest. For example, anti-CD63 conjugated-beads could be used to obtain CD63-enriched EVs, but effects of other vesicles may be neglected during subsequent experiments. This may explain why several metastasis and drug resistanceassociated proteins were not highly expressed in purified sEVs obtained via IAC. However, future basic and clinical studies are likely to provide valuable information regarding their heterogeneity and advance our understanding of biological functions, thus reveal and harness their potentials for disease detection and therapy.

\section{Conclusions}

Current methods are useful for isolating sEVs. Recommendations for choosing sEVs isolating method depend on the aim of study. For isolation of Panc-1 cell-derived sEVs, UC was advised to concentrate the medium. For purification, DGUC method could obtain high amount of sEVs particles with controlled size, IAC method is effective for isolating sEVs with high purity, but may loss subtypes of sEVs via specific marker capturing.

\section{Acknowledgements}

The authors would like to thank all staff of the Center for Medical Research, the Second Xiangya Hospital of Central South University.

\section{Funding}

This work was supported by Hunan Provincial Science and Technology Plan (2016TP2002) 


\section{Availability of data and materials}

Not applicable.

\section{Ethics approval and consent to participate}

Not applicable.

\section{Consent for publication}

Not applicable.

\section{Conflict of interests}

The authors declare that they have no competing interests.

\section{Author details}

${ }^{1}$ Department of Pharmacy, The Second Xiangya Hospital, Central South University, Changsha, Hunan, China. ${ }^{2}$ Hunan Provincial Engineering Research Centre of Translational Medicine and Innovative Drug, Changsha, Hunan, China. ${ }^{3}$ Institute of Clinical Pharmacy, Central South University, Changsha, Hunan, China.

Received: 16 November 2020 Accepted: 4 February 2021 Published online: 10 February 2021

\section{References}

1. Lee YXF, Johansson H, Wood MJA, El Andaloussi S. Considerations and implications in the purification of extracellular vesicles-a cautionary tale. Front Neurosci. 2019;13:1067.

2. Cheng $H$, Fang $H, X u R D$, et al. Development of a rinsing separation method for exosome isolation and comparison to conventional methods. Eur Rev Med Pharmacol Sci. 2019:23(12):5074-83.

3. Chen D, Wu X, Xia M, et al. Upregulated exosomic miR23b3p plays regulatory roles in the progression of pancreatic cancer. Oncol Rep. 2017;38(4):2182-8.

4. Han S, Huo Z, Nguyen K, et al. The proteome of pancreatic cancer-derived exosomes reveals signatures rich in key signaling pathways. Proteomics. 2019;19(13):e1800394.

5. Patel GK, Khan MA, Zubair H, et al. Comparative analysis of exosome isolation methods using culture supernatant for optimum yield, purity and downstream applications. Sci Rep. 2019;9(1):5335.

6. Whittaker TE, Nagelkerke A, Nele V, Kauscher U, Stevens MM. Experimental artefacts can lead to misattribution of bioactivity from soluble mesenchymal stem cell paracrine factors to extracellular vesicles. J Extracell Vesicles. 2020;9(1):1807674

7. Thery C, Witwer KW, Aikawa E, et al. Minimal information for studies of extracellular vesicles 2018 (MISEV2018): a position statement of the International Society for Extracellular Vesicles and update of the MISEV2014 guidelines. J Extracell Vesicles. 2018;7(1):1535750.

8. Doyle LM, Wang MZ. Overview of extracellular vesicles, their origin, composition, purpose, and methods for exosome isolation and analysis. Cells. 2019;8(7):727.

9. Duong P, Chung A, Bouchareychas L, Raffai RL. Cushioned-Density Gradient Ultracentrifugation (C-DGUC) improves the isolation efficiency of extracellular vesicles. PLoS One. 2019;14(4):e0215324.

10. Shao H, Im H, Castro CM, Breakefield X, Weissleder R, Lee H. New technologies for analysis of extracellular vesicles. Chem Rev. 2018;1 18(4):1917-50.

11. Li P, Kaslan M, Lee SH, Yao J, Gao Z. Progress in exosome isolation techniques. Theranostics. 2017;7(3):789-804

12. Monguio-Tortajada M, Galvez-Monton C, Bayes-Genis A, Roura S, Borras FE. Extracellular vesicle isolation methods: rising impact of size-exclusion chromatography. Cell Mol Life Sci. 2019;76(12):2369-82.

13. Hou R, Li Y, Sui Z, et al. Advances in exosome isolation methods and their applications in proteomic analysis of biological samples. Anal Bioanal Chem. 2019:411(21):5351-61.

14. Mitchell PJ, Welton J, Staffurth J, et al. Can urinary exosomes act as treatment response markers in prostate cancer? J Transl Med. 2009;7:4.

15. Van Deun J, Mestdagh P, Sormunen R, et al. The impact of disparate isolation methods for extracellular vesicles on downstream RNA profiling. J Extracell Vesicles. 2014:3·24858.
16. Ha D, Yang N, Nadithe V. Exosomes as therapeutic drug carriers and delivery vehicles across biological membranes: current perspectives and future challenges. Acta Pharm Sin B. 2016;6(4):287-96.

17. Melo SA, Luecke LB, Kahlert C, et al. Glypican-1 identifies cancer exosomes and detects early pancreatic cancer. Nature. 2015;523(7559):177-82

18. Li YJ, Wu JY, Wang JM, Hu XB, Cai JX, Xiang DX. Gemcitabine loaded autologous exosomes for effective and safe chemotherapy of pancreatic cancer. Acta Biomater. 2020;101:519-30.

19. Raza A, Ki CS, Lin CC. The influence of matrix properties on growth and morphogenesis of human pancreatic ductal epithelial cells in 3D. Biomaterials. 2013;34(21):5117-27.

20. Li Y-J, Wu J-Y, Wang J-M, Hu X-B, Cai J-X, Xiang D-X. Gemcitabine loaded autologous exosomes for effective and safe chemotherapy of pancreatic cancer. Acta Biomaterialia. 2019;101:519.

21. Thery C, Amigorena S, Raposo G, Clayton A. Isolation and characterization of exosomes from cell culture supernatants and biological fluids. Curr Protoc Cell Biol. 2006; Chap. 3:Unit 322.

22. Lobb RJ, Becker M, Wen SW, et al. Optimized exosome isolation protocol for cell culture supernatant and human plasma. J Extracell Vesicles. 2015:4:27031.

23. Paolini L, Zendrini A, Di Noto G, et al. Residual matrix from different separation techniques impacts exosome biological activity. Sci Rep. 2016;6:23550.

24. Boing AN, van der Pol E, Grootemaat AE, Coumans FA, Sturk A, Nieuwland $R$. Single-step isolation of extracellular vesicles by size-exclusion chromatography. J Extracell Vesicles. 2014:3:23430.

25. Koh YQ, Almughlliq FB, Vaswani K, Peiris HN, Mitchell MD. Exosome enrichment by ultracentrifugation and size exclusion chromatography. Front Biosci (Landmark Ed). 2018;23:865-74.

26. Cui Y, Tian M, Zong M, et al. Proteomic analysis of pancreatic ductal adenocarcinoma compared with normal adjacent pancreatic tissue and pancreatic benign cystadenoma. Pancreatology. 2009;9(1-2):89-98.

27. Cui $Y$, Wu J, Zong M, et al. Proteomic profiling in pancreatic cancer with and without lymph node metastasis. Int J Cancer. 2009;124(7):1614-21.

28. Le Large TYS, El Hassouni B, Funel N, et al. Proteomic analysis of gemcitabine-resistant pancreatic cancer cells reveals that microtubuleassociated protein 2 upregulation associates with taxane treatment. Therapeutic Adv Med Oncol. 2019;11:1758835919841233.

29. Yang Y, Boza-Serrano A, Dunning CJR, Clausen BH, Lambertsen KL, Deierborg T. Inflammation leads to distinct populations of extracellular vesicles from microglia. J Neuroinflammation. 2018;15(1):168.

30. Zhu X, Shen H, Yin X, et al. Macrophages derived exosomes deliver miR-223 to epithelial ovarian cancer cells to elicit a chemoresistant phenotype. J Exp Clin Cancer Res. 2019;38(1):81.

31. Chen CW, Wang LL, Zaman S, et al. Sustained release of endothelial progenitor cell-derived extracellular vesicles from shear-thinning hydrogels improves angiogenesis and promotes function after myocardial infarction. Cardiovasc Res. 2018;114(7):1029-40.

32. Hoshyar N, Gray S, Han H, Bao G. The effect of nanoparticle size on in vivo pharmacokinetics and cellular interaction. Nanomedicine. 2016;11(6):673-92.

33. Caponnetto F, Manini I, Skrap M, et al. Size-dependent cellular uptake of exosomes. Nanomedicine. 2017;13(3):1011-20.

34. Masyuk Al, Huang BQ, Ward CJ, et al. Biliary exosomes influence cholangiocyte regulatory mechanisms and proliferation through interaction with primary cilia. Am J Physiol Gastrointest Liver Physiol. 2010;299(4):G990-9.

35. Oksvold MP, Neurauter A, Pedersen KW. Magnetic bead-based isolation of exosomes. Methods Mol Biol. 2015;1218:465-81.

36. Huang L, Wang DB, Singh $N$, Yang F, Gu N, Zhang XE. A dual-signal amplification platform for sensitive fluorescence biosensing of leukemiaderived exosomes. Nanoscale. 2018;10(43):20289-95.

37. Baranyai T, Herczeg K, Onodi Z, et al. Isolation of exosomes from blood plasma: qualitative and quantitative comparison of ultracentrifugation and size exclusion chromatography methods. PLoS One. 2015:10(12):e0145686.

38. Barranco I, Padilla L, Parrilla I, et al. Extracellular vesicles isolated from porcine seminal plasma exhibit different tetraspanin expression profiles. Sci Rep. 2019:9(1):11584

39. Kang YT, Purcell E, Palacios-Rolston C, et al. Isolation and profiling of circulating tumor-associated exosomes using extracellular 
vesicular lipid-protein binding affinity based microfluidic device. Small. 2019;15(47):e1903600.

40. Tian Y, Gong M, Hu Y, et al. Quality and efficiency assessment of six extracellular vesicle isolation methods by nano-flow cytometry. J Extracell Vesicles. 2020;9(1):1697028.

41. Yang F, Liao X, Tian Y, Li G. Exosome separation using microfluidic systems: size-based, immunoaffinity-based and dynamic methodologies. Biotechnol J. 2017;12(4):1600699.

42. Contreras-Naranjo JC, Wu HJ, Ugaz VM. Microfluidics for exosome isolation and analysis: enabling liquid biopsy for personalized medicine. Lab Chip. 2017;17(21):3558-77.

43. Charoenviriyakul C, Takahashi Y, Nishikawa M, Takakura Y. Preservation of exosomes at room temperature using lyophilization. Int J Pharm. 2018;553(1-2):1-7.

44. Purushothaman A. Exosomes from cell culture-conditioned medium: isolation by ultracentrifugation and characterization. Methods Mol Biol. 2019;1952:233-44.

45. Cai S, Luo B, Jiang P, et al. Immuno-modified superparamagnetic nanoparticles via host-guest interactions for high-purity capture and mild release of exosomes. Nanoscale. 2018;10(29):14280-9.
46. Peng Q, Zhang J, Zhou G. Comparison of plasma exosomes by differential ultracentrifugation and solvent precipitation methods. Clin Lab. 2018;64(6):991-8

47. Cao F, Gao Y, Chu Q, et al. Proteomics comparison of exosomes from serum and plasma between ultracentrifugation and polymer-based precipitation kit methods. Electrophoresis. 2019;40(23-24):3092-8.

48. Jeppesen DK, Fenix AM, Franklin JL, et al. Reassessment of exosome composition. Cell. 2019;177(2):428-45 e418.

49. Campoy I, Lanau L, Altadill T, et al. Exosome-like vesicles in uterine aspirates: a comparison of ultracentrifugation-based isolation protocols. J Transl Med. 2016;14(1):180.

50. Kalluri R, LeBleu VS. The biology, function, and biomedical applications of exosomes. Science. 2020;367(6478).

\section{Publisher's Note}

Springer Nature remains neutral with regard to jurisdictional claims in published maps and institutional affiliations.
Ready to submit your research? Choose BMC and benefit from:

- fast, convenient online submission

- thorough peer review by experienced researchers in your field

- rapid publication on acceptance

- support for research data, including large and complex data types

- gold Open Access which fosters wider collaboration and increased citations

- maximum visibility for your research: over $100 \mathrm{M}$ website views per year

At BMC, research is always in progress.

Learn more biomedcentral.com/submissions 